\title{
A study of an implementation method for making a synchronised schedule of the mass transit network
}

\author{
K. Moro \& Y. Sugai \\ Graduate School of Engineering, Chiba University, Japan
}

\begin{abstract}
In this paper we propose the concept of entropy of the timetable to evaluate the mass transit system. This concept simplifies the problem of the synchronisation of the timetable. Additionally, we show how to use the concept of the entropy for evaluation. We first describe the importance of the synchronised periodic railway timetable, and also the conditions for the synchronisation of the timetable. We next present a difficulty encountered when we synchronise the timetable completely everywhere in the transit network. This difficulty is attributed to the restriction of train headway in the synchronisation of the timetable. Then we must simplify the problem of synchronisation of the timetable by removing some constraints. Lastly, we indicate that using the concept of the entropy is useful for solving the complex problem.
\end{abstract}

Keywords: entropy, synchronisation of timetable, periodic timetable, evaluate mass transit system.

\section{Introduction}

In mass transit systems such as railway networks, there are a large amount of passengers, and OD-pair passengers travelling in a huge number of combinations. Even in the demand of the same OD-pair, it is common that there are various times when the passengers wish to start and end their travelling. In order to strictly manage all such demands for the travelling, a huge number of trains need to operate. However the capacity of the tracks is limited, and the number of trains required exceeds this capacity. In addition, the number of passengers on each train often becomes too small to operate a train efficiently. Vuchic [1] analysed 
the topology of the railway network in the mega cities from the point of view of solving the capacity shortage of the infrastructure.

Actually, many mass transit systems are managed by a small number of service routes. The number of service routes is small when compared with the number of varieties for demand. By limiting the number of service routes, the passengers of various OD-pairs have to board trains together. Moreover, most of the passengers must select a scheduled train which is permitted by the passenger for travelling. That is, the passengers are limited in their behaviour for travelling by the timetable of trains.

However, the operator can manage the huge demand for travelling by limiting the number of service routes and trains, in the case that the capacity of the trains is large enough and the schedule of trains is appropriate. Furthermore, decreasing the number of trains leads to avoiding excess capacity on the tracks. It is ideal that many passengers can travel at the time they desire and have fewer detours caused by the provided transit service. Whereas, by limiting the service route, many passengers will have to change trains at some junctions to arrive at their destination. Therefore, it is important to decrease the waiting time at junctions as much as possible for passengers. This requirement causes an additional constraint for the timetabling problem. Adding this "connection constraint" causes the solution for timetabling problems to become more difficult to find. If a disruption occurs in the railway network with a completely synchronised timetable, the propagation of disruption becomes a serious problem [2].

In this paper, we consider the synchronisation of the timetable. First we will show some examples of the synchronisation of the timetable and the tight constraints for synchronisation. Secondly we will consider the case that the timetable is synchronised with little incompletion. Then we propose to use the concept of the entropy of the information theory to evaluate the level of synchronisation.

\section{Synchronisation of timetable}

In this section we describe the synchronisation of the timetable.

\subsection{Definition}

The synchronised timetable is the state in which the scheduled transfer time to the succeeding trains is not to long.

It is desirable to realise the synchronisation of the timetable at most of the junctions.

\subsection{Importance of the synchronised timetable}

When the timetable of the transit network is synchronised, we can reduce the stagnant time of the passengers who transfer trains at junctions. Reducing the stagnant time at the junctions means reducing the unnecessary time for the 
passengers. Moreover the travelling time of the passengers is reduced. This is desirable for the passengers, and it is a great pleasure, not only the passengers but also for the operators of the transit systems. The reason for this is because the operators can manage the demands of passengers well, while using less rolling stock and less crew.

\subsection{Example at a junction}

We introduce the example of the timetable at Bern in Switzerland and we show that the timetable of "Inter City" and "Inter Ragio" at Bern is synchronised.

\subsubsection{Synchronisation at Bern}

The departure and arrival times at Bern station is as shown in Figure 1. The arrows towards the circumference from the interior of the circle show the arrival times of the trains. The arrows towards from the circumference of the circle to the outside show the departure times of the trains. The arrows with dashed tails describe the trains from/to east area (e.g. Zürich, Olten and Luzern). The arrows with solid tails describe the trains from/to south and west area (e.g. Spiez and Lausanne). All passenger transfer trains are described with different types of arrows.

We recognise that there are two groups of arrivals and departures. One group of arrows touches the circumference near the top the circle. The other one touches the circumference near the bottom the circle.

Within each group, the transfer time for trains within the same group is less than 20 minutes. Furthermore, the transfer time between the two groups is never more than 4 minutes. This shows that the passengers can transfer trains in the same group of this figure. Thus we can say that the timetable at Bern is synchronised.

\subsection{A case of the cyclic route}

We consider the synchronisation of the timetable of the cyclic route. And we assume that the period of the integrated timetable is $h$ and the necessary time travelling round this circuit is $r$.

If equation (1) is satisfied, we can say the timetable is synchronised everywhere in the cyclic route.

$$
\exists k \in \mathbb{N}^{+}, h \gg \delta \geq 0 \text { s.t. } r+\delta=k \cdot h .
$$

$\mathbb{N}^{+}$describes the set of nonnegative integers. In addition, we can say that the timetable is synchronised enough when the transfer time at each junction is similar to or less than $\delta$.

\subsubsection{Example}

Take the circuit of the railway network that consists, for example, of the links: Bern - Visp - Lausanne - Bern. When travelling on this circuit, we must transfer at Bern, Visp and Lausanne when we pass. 


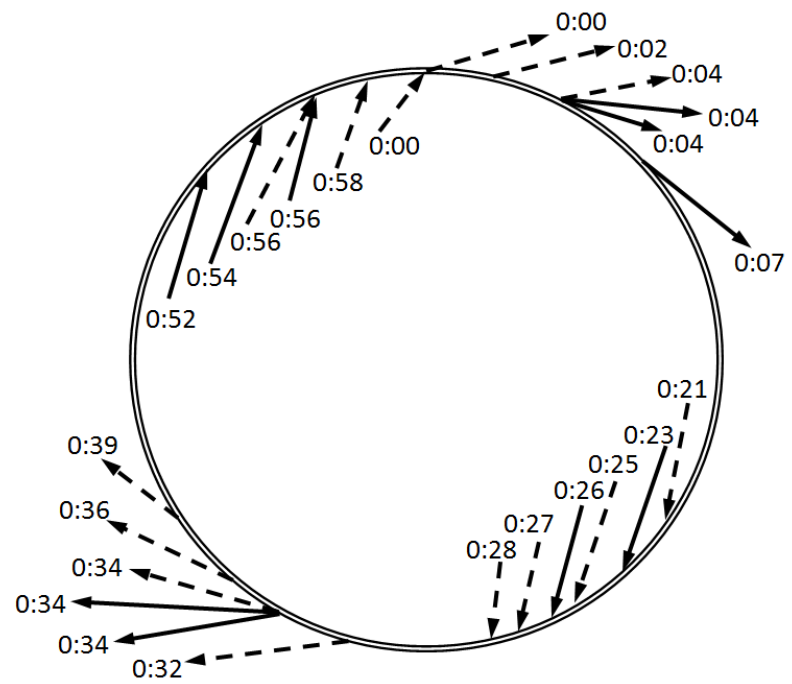

Figure 1: Configuration of connection at Bern.

There exists the trains operated by the timetable as in Table 1 every hour. The period of the clockwise route is $h_{c}=60$ and the necessary time is $r_{c}=229$. The period of the anticlockwise route is $h_{a c}=60$ and the necessary time is $r_{a c}=230$ similarly. The maximum transfer time on the clockwise route is 11 minutes, and on the anticlockwise route it is 10 minutes.

Table 1: Transfer timetable of circuit.

\begin{tabular}{|c|c|c|c|c|c|c|}
\hline \multicolumn{7}{|c|}{ Clockwise } \\
\hline Bern & $\rightarrow$ & Visp & $\rightarrow$ & Lausanne & $\rightarrow$ & Bern \\
\hline $0: 07$ & & 1:02/1:06 & & $2: 40 / 2: 50$ & & $3: 56 /(0: 07)$ \\
\hline \multicolumn{7}{|c|}{ Anti-clockwise } \\
\hline Bern & $\rightarrow$ & Lausanne & $\rightarrow$ & Visp & $\rightarrow$ & Bern \\
\hline $0: 04$ & & $1: 10 / 1: 20$ & & $2: 53 / 2: 57$ & & $3: 54 /(0: 04)$ \\
\hline
\end{tabular}

Then $\delta$ and $k$ satisfy the conditional expression (1) are as in Table 2. And the transfer time in each direction is equal to $\delta$. Thus we can say that the timetable of this example is synchronised. 
Table 2: Values: $\delta$ and $k$.

\begin{tabular}{|c|cc|}
\hline Direction & $\delta$ & $k$ \\
\hline Clockwise & 11 & 4 \\
Anti-clockwise & 10 & 4 \\
\hline
\end{tabular}

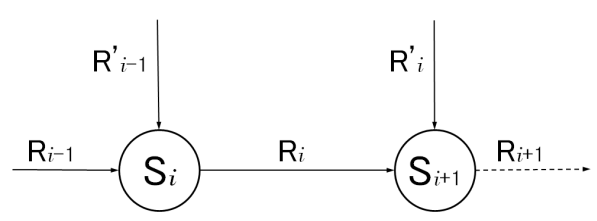

Figure 2: Example of a network.

\subsection{A case of multiple merging}

We consider the synchronisation of timetable when merging a route with another continuously.

\subsubsection{Topology of network}

We consider the configuration of railway network as in Figure 2. Two routes which are $R_{i-1}$ and $R_{i-1}^{\prime}$, come into $S_{i}$. And one route which is $R_{i}$, goes out from $S_{i}$.

\subsubsection{Timetable}

We assume the timetable is as follows.

- Trains running on $R_{i}$ are operated by the periodic timetable $T_{i}$.

- Headway of $R_{i}$ is $h_{i}$.

- Arrival time to $S_{i}$ of a train running on $R_{i-1}$ is $a_{i-1}$.

- Departure time from $S_{i}$ of a train running on $R_{i}$ is $d_{i}$.

Let $\tau_{i}$ be the set of $d_{i}$. Let $\hat{d}_{i}$ be the departure time of the train connecting from $a_{i-1}$ at $S_{i}$, i.e.

$$
\begin{aligned}
\hat{d}_{i} & =\arg \min _{d_{i} \in \tau_{i}}\left(d_{i}-a_{i-1}\right), \\
\hat{d}^{\prime} & =\arg \min _{d_{i} \in \tau_{i}}\left(d_{i}-a_{i-1}^{\prime}\right) .
\end{aligned}
$$

Each predecessor train connects with a distinct successor train when the following conditions are satisfied,

$$
\begin{aligned}
& 0 \leq \hat{d}_{i}-a_{i-1} \leq h_{i-1}, \\
& 0 \leq \hat{d}_{i}^{\prime}-a_{i-1} \leq h_{i-1}^{\prime} .
\end{aligned}
$$

From the definition of $\hat{d}_{i}$ and $\hat{d}^{\prime}{ }_{i}$, the following conditions are satisfied,

$$
\hat{d}_{i}-h_{i}-a_{i-1}<0 \Rightarrow \hat{d}_{i}-a_{i-1}<h_{i},
$$




$$
\hat{d}_{i}^{\prime}-h_{i}-a_{i-1}^{\prime}<0 \Rightarrow \hat{d}_{i}^{\prime}-a_{i-1}^{\prime}<h_{i}
$$

Furthermore the following conditions of headway are satisfied:

$$
\begin{aligned}
& h_{i} \leq h_{i-1}, \\
& h_{i} \leq h_{i-1}^{\prime} .
\end{aligned}
$$

Then we consider the headway as a sequence indexed by $i, h_{i}$ as the monotonically decreasing sequence.

\subsubsection{Conclusion of the case of multiple merging}

It is necessary that the headway of the later successor is small enough to synchronise with the predecessor. In other words, when the synchronisations are iterated many times, the headway of the later successor becomes too small.

\section{Entropy of timetable}

It often happens that the perfect synchronisation of the timetable is difficult. It is especially difficult in the case when some routes arrive and depart by a different headway to each other at the junction.

We suppose the useful concept which is the entropy of the timetable. The concept of the entropy of the information theory is useful to evaluate the convenience of the timetable of transport networks [3]. Using the concept of the entropy, we can measure the level of synchronisation of the timetable.

\subsection{Definition}

\subsubsection{Shannon's entropy of the information theory}

Let $X$ be a discrete random variable on a finite set $X=\left\{x_{1}, \ldots, x_{n}\right\}$, with probability distribution function $p(x):=\operatorname{Pr}(X=x)$. The entropy $H(X)$ of $X$ is defined as

$$
H(X)=-\sum_{x \in X} p(x) \cdot \log p(x) .
$$

The convention $0 \log 0=0$ is adopted in the definition. The logarithm is usually taken to the base 2 , in which case the entropy is measured in "bits".

\subsubsection{Probability distribution function of the timetable}

Let $\mathcal{T}=\left\{T r_{1}, T r_{2}, \ldots, T r_{n}\right\}$ be the set of trains for travelling between the specific origin and the specific destination. Let $b_{i}$ be the number of passengers boarding on the train $\operatorname{Tr}_{i}$.

We define the probability distribution function $p_{i}$ as

$$
p_{i}=\frac{b_{i}}{\sum_{T r_{j} \in \mathcal{T}} b_{j}} .
$$

If the passengers have to transfer the trains at the intermediate stations, $\operatorname{Tr}_{i}$ is a "train set" [3]. 


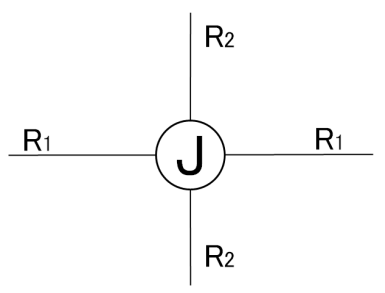

Figure 3: Example of the junction.

\subsection{Properties of the entropy}

As the passengers are equally distributed on each train, the entropy increases.

\subsection{Examples}

We consider the junction of the crossing two routes as shown in Figure 3.

\subsubsection{Timetable}

Each route is operated by the periodic timetable at regular intervals.

We denote $f_{i}$ as the number of trains per hour of the route $R_{i}$. We consider the timetable of each frequency at $J$, that is shown in Table 3 . This timetable expresses the arrival and departure of the $j$ th train at the column $(j)$ in the cases of some frequencies: $12,10,6,5,4,3$ and 2 .

\subsubsection{Passengers}

We consider some cases in which we calculate the entropies. All passengers travel to $J$ by the train running on the route $R_{1}$ and transfer to the train on the route $R_{2}$ at $J$.

We assume the behaviour of the passengers as follows:

(1) Each passenger takes the train on the route $R_{1}$ by which the passenger is able to ensure the transfer time at $J$ is 1 to 6 minutes.

(2) All passengers come to the origin station according to a uniform distribution.

(3) The number of passengers is large enough.

The above condition (1) says that "the limit of transfer time" is 6 minutes.

\subsubsection{In the case of high frequency}

We consider the case of $f_{1}<f_{2}$. If the headway of the route $R_{2}$ exceeds the limit of transfer time, all trains on the route $R_{1}$ connect to the trains of $R_{2}$ in 6 minutes. Hence the entropy is

$$
H=\log f_{1} .
$$

Thus, we consider the case of $f_{2}<10$ in the following. 
Table 3: Timetable of $J$.

\begin{tabular}{|c|c|c|c|c|c|c|c|c|c|c|c|c|c|}
\hline$f_{i}$ & & (1) & (2) & (3) & (4) & (5) & (6) & (7) & (8) & (9) & (10) & (11) & (12) \\
\hline \multirow{2}{*}{$f_{i}=12$} & arr. & 59 & 04 & 09 & 14 & 19 & 24 & 29 & 34 & 39 & 44 & 49 & 54 \\
\hline & dep. & 00 & 05 & 10 & 15 & 20 & 25 & 30 & 35 & 40 & 45 & 50 & 55 \\
\hline \multirow{2}{*}{$f_{i}=10$} & arr. & 59 & 05 & 11 & 17 & 23 & 29 & 35 & 41 & 47 & 53 & - & - \\
\hline & dep. & 00 & 06 & 12 & 18 & 24 & 30 & 36 & 42 & 48 & 54 & - & - \\
\hline \multirow{2}{*}{$f_{i}=6$} & arr. & 59 & 09 & 19 & 29 & 39 & 49 & - & - & - & - & - & - \\
\hline & dep. & 00 & 10 & 20 & 30 & 40 & 50 & - & - & - & - & - & - \\
\hline \multirow{2}{*}{$f_{i}=5$} & arr. & 59 & 11 & 23 & 35 & 47 & - & - & - & - & - & - & - \\
\hline & dep. & 00 & 12 & 24 & 36 & 48 & - & - & - & - & - & - & - \\
\hline \multirow{2}{*}{$f_{i}=4$} & arr. & 59 & 14 & 29 & 44 & - & - & - & - & - & - & - & - \\
\hline & dep. & 00 & 15 & 30 & 45 & - & - & - & - & - & - & - & - \\
\hline \multirow{2}{*}{$f_{i}=3$} & arr. & 59 & 19 & 39 & - & - & - & - & - & - & - & - & - \\
\hline & dep. & 00 & 20 & 40 & - & - & - & - & - & - & - & - & - \\
\hline \multirow{2}{*}{$f_{i}=2$} & arr. & 59 & 29 & - & - & - & - & - & - & - & - & - & - \\
\hline & dep. & 00 & 30 & - & - & - & - & - & - & - & - & - & - \\
\hline
\end{tabular}

\subsubsection{The case: numbers of frequencies are relatively prime}

We consider the pairs of relatively prime numbers that consist of the combination of 3,4 and 5. The probabilities and the entropies of the example are shown in Table 4.

We describe the combined row of Table 4. As an example, " $k \rightarrow l$ " of the first column means the transfer from $R_{1}$, whose frequency is $k$ per hour, to $R_{2}$, whose

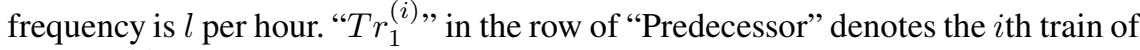
$R_{1}$. " $\operatorname{Tr}_{2}^{(j)}$ " in the row of "Successor" denotes the $j$ th train of $R_{2}$. "-" in the row of "Successor" denotes that the $\operatorname{Tr}_{1}^{(i)}$ above has no successor train of $R_{2}$ satisfies the passengers behaviour. The values in the row of $p_{i}$ denote the probabilities of the train set $\operatorname{Tr}_{1}^{(i)}$ and $\operatorname{Tr}_{2}^{(j)}$. The value of the column " $H$ " denotes the entropy of the timetable in the case of the transfer $k \rightarrow l$. 
Table 4: Entropies (relatively prime).

\begin{tabular}{|c|c|c|c|c|c|c|c|}
\hline$f_{1} \rightarrow f_{2}$ & & & & & & & $\mathrm{H}$ \\
\hline \multirow{3}{*}{$5 \rightarrow 4$} & Predecessor & $T r_{1}^{(1)}$ & $T r_{2}^{(1)}$ & $T r_{3}^{(1)}$ & $T r_{4}^{(1)}$ & $T r_{5}^{(1)}$ & \multirow{3}{*}{0.722} \\
\hline & Successor & $\rightarrow T r_{1}^{(2)}$ & $\rightarrow T r_{2}^{(2)}$ & - & - & - & \\
\hline & $p_{i}$ & $\frac{4}{5}$ & $\frac{1}{5}$ & 0 & 0 & 0 & \\
\hline \multirow{3}{*}{$5 \rightarrow 3$} & Predecessor & $T r_{1}^{(1)}$ & $T r_{2}^{(1)}$ & $T r_{3}^{(1)}$ & $\operatorname{Tr}_{4}^{(1)}$ & $T r_{5}^{(1)}$ & \multirow{3}{*}{0.971} \\
\hline & Successor & $\rightarrow T r_{1}^{(2)}$ & - & - & $\rightarrow T r_{3}^{(2)}$ & - & \\
\hline & $p_{i}$ & $\frac{2}{5}$ & 0 & 0 & $\frac{3}{5}$ & 0 & \\
\hline \multirow{3}{*}{$4 \rightarrow 5$} & Predecessor & $T r_{1}^{(1)}$ & $\operatorname{Tr}_{2}^{(1)}$ & $T r_{3}^{(1)}$ & $T r_{4}^{(1)}$ & & \multirow{3}{*}{0.811} \\
\hline & Successor & $\rightarrow T r_{1}^{(2)}$ & - & - & $\rightarrow T r_{5}^{(2)}$ & & \\
\hline & $p_{i}$ & $\frac{1}{4}$ & 0 & 0 & $\frac{3}{4}$ & & \\
\hline \multirow{3}{*}{$4 \rightarrow 3$} & Predecessor & $T r_{1}^{(1)}$ & $T r_{2}^{(1)}$ & $T r_{3}^{(1)}$ & $T r_{4}^{(1)}$ & & \multirow{3}{*}{0.811} \\
\hline & Successor & $\rightarrow T r_{1}^{(2)}$ & $\rightarrow T r_{2}^{(2)}$ & - & - & & \\
\hline & $p_{i}$ & $\frac{3}{4}$ & $\frac{1}{4}$ & 0 & 0 & & \\
\hline \multirow{3}{*}{$3 \rightarrow 5$} & Predecessor & $T r_{1}^{(1)}$ & $T r_{2}^{(1)}$ & $T r_{3}^{(1)}$ & & & \multirow{3}{*}{0.918} \\
\hline & Successor & $\rightarrow \operatorname{Tr}_{1}^{(2)}$ & $\rightarrow T r_{3}^{(2)}$ & - & & & \\
\hline & $p_{i}$ & $\frac{2}{3}$ & $\frac{1}{3}$ & 0 & & & \\
\hline \multirow{3}{*}{$3 \rightarrow 4$} & Predecessor & $T r_{1}^{(1)}$ & $T r_{2}^{(1)}$ & $T r_{3}^{(1)}$ & & & \multirow{3}{*}{0.918} \\
\hline & Successor & $\rightarrow T r_{1}^{(2)}$ & - & $\rightarrow \operatorname{Tr}_{4}^{(2)}$ & & & \\
\hline & $p_{i}$ & $\frac{1}{3}$ & 0 & $\frac{2}{3}$ & & & \\
\hline
\end{tabular}

\subsubsection{The case: numbers of frequencies are not relatively prime.}

We consider the case that the numbers of frequencies per hour are not relatively prime. Let $f_{c}$ be the greatest common divisor of $f_{1}$ and $f_{2}$. The departure time of every $\left(f_{i} / f_{c}\right)$ th train on the route $R_{i}$ is the same as the departure time of a train on the other route. Therefore the probability of every $\left(f_{i} / f_{c}\right)$ th train is not zero.

We consider the pairs of not relatively prime numbers that consist of the combination of 4,6 and 10. The probabilities and the entropies of the example is shown in Table 5 and has the same notation as Table 4. 
Table 5: Entropies (not relatively prime).

\begin{tabular}{|c|c|c|c|c|c|c|c|}
\hline$f_{1} \rightarrow f_{2}$ & & & & & & & $\mathrm{H}$ \\
\hline \multirow{6}{*}{$10 \rightarrow 6$} & Predecessor & $T r_{1}^{(1)}$ & $\operatorname{Tr}_{2}^{(1)}$ & $T r_{3}^{(1)}$ & $T r_{4}^{(1)}$ & $T r_{5}^{(1)}$ & \multirow{6}{*}{1.995} \\
\hline & Successor & $\rightarrow \operatorname{Tr}_{1}^{(2)}$ & $\rightarrow \operatorname{Tr}_{2}^{(2)}$ & - & $\rightarrow \operatorname{Tr}_{3}^{(2)}$ & - & \\
\hline & $p_{i}$ & $\frac{2}{10}$ & $\frac{1}{10}$ & 0 & $\frac{2}{10}$ & 0 & \\
\hline & Predecessor & $T r_{6}^{(1)}$ & $\operatorname{Tr}_{7}^{(1)}$ & $T r_{8}^{(1)}$ & $T r_{9}^{(1)}$ & $T r_{10}^{(1)}$ & \\
\hline & Successor & $\rightarrow T r_{4}^{(2)}$ & $\rightarrow \operatorname{Tr}_{5}^{(2)}$ & - & $\rightarrow \operatorname{Tr}_{6}^{(2)}$ & - & \\
\hline & $p_{i}$ & $\frac{2}{10}$ & $\frac{1}{10}$ & 0 & $\frac{2}{10}$ & 0 & \\
\hline \multirow{6}{*}{$10 \rightarrow 4$} & Predecessor & $T r_{1}^{(1)}$ & $\operatorname{Tr}_{2}^{(1)}$ & $T r_{3}^{(1)}$ & $T r_{4}^{(1)}$ & $T r_{5}^{(1)}$ & \multirow{6}{*}{1.971} \\
\hline & Successor & $\rightarrow \operatorname{Tr}_{1}^{(2)}$ & - & $\rightarrow \operatorname{Tr}_{2}^{(2)}$ & - & - & \\
\hline & $p_{i}$ & $\frac{3}{10}$ & 0 & $\frac{2}{10}$ & 0 & 0 & \\
\hline & Predecessor & $\operatorname{Tr}_{6}^{(1)}$ & $\operatorname{Tr}_{7}^{(1)}$ & $\operatorname{Tr}_{8}^{(1)}$ & $\operatorname{Tr}_{9}^{(1)}$ & $T r_{10}^{(1)}$ & \\
\hline & Successor & $\rightarrow \operatorname{Tr}_{3}^{(2)}$ & - & $\rightarrow \operatorname{Tr}_{4}^{(2)}$ & - & - & \\
\hline & $p_{i}$ & $\frac{3}{10}$ & 0 & $\frac{2}{10}$ & 0 & 0 & \\
\hline \multirow{6}{*}{$6 \rightarrow 4$} & Predecessor & $T r_{1}^{(1)}$ & $\operatorname{Tr}_{2}^{(1)}$ & $T r_{3}^{(1)}$ & $T r_{4}^{(1)}$ & $T r_{5}^{(1)}$ & \multirow{6}{*}{1.918} \\
\hline & Successor & $\rightarrow T r_{1}^{(2)}$ & $\rightarrow \operatorname{Tr}_{2}^{(2)}$ & - & $\rightarrow \operatorname{Tr}_{3}^{(2)}$ & $\rightarrow \operatorname{Tr}_{4}^{(2)}$ & \\
\hline & $p_{i}$ & $\frac{2}{6}$ & $\frac{1}{6}$ & 0 & $\frac{2}{6}$ & $\frac{1}{6}$ & \\
\hline & Predecessor & $T r_{6}^{(1)}$ & & & & & \\
\hline & Successor & - & & & & & \\
\hline & $p_{i}$ & 0 & & & & & \\
\hline \multirow{3}{*}{$4 \rightarrow 6$} & Predecessor & $T r_{1}^{(1)}$ & $T r_{2}^{(1)}$ & $T r_{3}^{(1)}$ & $\overline{T r_{4}^{(1)}}$ & & \multirow{3}{*}{2.000} \\
\hline & Successor & $\rightarrow T r_{1}^{(2)}$ & $\rightarrow \mathrm{Tr}_{3}^{(2)}$ & $\rightarrow T r_{4}^{(2)}$ & $\rightarrow \operatorname{Tr}_{6}^{(2)}$ & & \\
\hline & $p_{i}$ & $\frac{1}{4}$ & $\frac{1}{4}$ & $\frac{1}{4}$ & $\frac{1}{4}$ & & \\
\hline
\end{tabular}

\subsection{Conclusion of the entropy}

We considered the case of the travelling path with transfers at intermediate junctions. Then we obtained the following result. The entropy of the timetable 
is inversely proportional to the amount of transfer time which the passengers need to travel by the timetable. As a consequence, the timetable in which the entropy is large, is synchronised well.

\section{Conclusions}

In this paper we describe the ways to implement the making of a synchronised timetable. One way of implementing it is the case of the cyclic route. The other is in the case with multiple merging.

However, sometimes we cannot make a timetable which is completely synchronised. In this case we substitute a timetable which is roughly synchronised. Therefore we proposed a method for evaluating the level of synchronisation in the timetable. The way used is the concept of the entropy of the information theory. We show that the concept of the entropy is one of the most useful methods to measure the level of synchronisation of the timetable.

In conclusion, the concept of the entropy is a useful and simple method to evaluate the quality of the synchronised timetable. Furthermore, calculating the entropy is the easiest way to evaluate the quality of the transit system.

\section{References}

[1] Vuchic, V.R., Planning, design and operation of rail transit networks. Metro Report International, 2014 (December), pp. 48-53, 2014.

[2] Heidergott, B., Olsder, G.J. \& van der Woude, J., Max Plus at work: modeling and analysis of synchronized systems: a course on Max-Plus algebra and its applications. Princeton University Press, 2006.

[3] Moro, K. \& Sugai, Y., A relation between a robustness of the disruption of the train schedule and the entropy of information theory. RailTokyo2015 (to appear). 